\title{
Playing with the Sun: A Virtual Physics Experience for Nuclear Fusion Experimentation and Learning
}

\author{
Helena Lennholm \\ Eike Falk Anderson \\ The National Centre for Computer Animation \\ Bournemouth University, United Kingdom \\ Email: hel.lennholm@hotmail.co.uk_ eikea@siggraph.org
}

\begin{abstract}
Nuclear fusion has the potential to become an important energy source in the future, so we propose a virtual physics experience for learning about nuclear fusion and plasma physics with an interactive fusion experiment, and then placing the user inside the experiment and letting them run and control this. Implemented using Unreal Engine 4, users can manipulate parameters of the experiment in real-time with immediate visual results in the form of the shape and colour glow of the virtual plasma, resembling that of a real plasma.

Index Terms-virtual physics experience, computer games technology, edutainment, nuclear fusion, tokamak
\end{abstract}

\section{INTRODUCTION}

Computer Games, Virtual Reality (VR) and Augmented Reality (AR), among other things, have the power to allow people to experience diverse domains such as history, art, science, etc. in new ways, bringing these close to them, independent of whether one is a student of the subject or simply an interested lay person. Thermonuclear fusion, the power of the sun, is one candidate for future power generation, and we proposed a 3D game-like application, a virtual physics experience (VPE), for bringing the world of nuclear fusion to audiences by employing an immersive approach, allowing users to 'play around' with the 'plasma' - fully ionized gas created inside a fusion reactor - and then see what happens. Users take on the role of the scientists in charge of running the fusion experiment, meaning they control the plasma, while being able to move around inside the device, learning about nuclear fusion and what this involves. Although the aim of our VPE is to create an educational interactive experience rather than a game, educational games and training systems have been used for inspiration and guidance.

\section{Nuclear Physics, Simulations \& Games}

Since the start of research into the possibility of creating a thermonuclear fusion reactor, there have been different approaches to the design (heating, plasma confinement, etc.) of reactors [1], the toroidal tokamak device [2] being one of the most researched of these, with the spherical tokamak currently being considered to be the most promising design for future energy production by nuclear physicists [3]. Educating students and the public on this technology is therefore important

There are many examples for serious games - both $2 \mathrm{D}$ and 3D - and 3D virtual environments being used for physics education. For example, just to name a few, Stege et al. used a serious game that had been developed by Eindhoven University of Technology "to instruct high-school students on electrical engineering" [4], Carr et al. created a version of the classic arcade game Asteroids that employs a relativistic physics engine, providing different game modes, both emulating the classical Asteroids by calculating "the motions of objects under the laws of Classical mechanics, and the other with the laws of Relativistic mechanics" to teach Einstein's Theory of Relativity [5], and Lin and Chiou modified a commercial game to convey Newton's Second Law of Motion [6]. Du et al. created a sandbox-game-like virtual environment for simple motion and collision experiments [7], and Hu et al. created a virtual game environment hosting electromagnetism experiments [8]. VR was used for the KATRIN particle physics experience to provide users with "a realistic impression of the real size of the experiment" [9].

There are fewer examples of educational games or virtual experiences concerned with nuclear physics, though. These range from simple interactive web-simulations or games, such as the Dalton Institute's "Nuclear Reactor Simulator" [10], which restricts users to a limited set of inputs to control the simulation (reactor core control rod positioning and steam generator output), to more complex VR simulations that provide detailed virtual replicas of university laboratory equipment [11]. Other examples take the shape of interactive e-learning modules in Learning Management Systems, such as those presented by Stanescu [12], although many of these, such as the former, tend to be nuclear safety training simulations, some of which also use VR [13].

An even smaller number of physics 'games' or simulations are actually concerned with nuclear fusion, such as Cham's interactive fusion "explainers" [14], and there also exist fusion reactor simulations that, similar to our proposed VPE, also employ the control of the plasma as their main interaction method. First, there is 'Operation Tokamak' [15], a simple (2D) mobile game created for EUROfusion, the European Consortium for the Development of Fusion Energy, which allows players to take control of a tokamak fusion reactor by manipulating two inputs, i.e. the strength of the magnetic field and the output of the heating system. Then there is 'Virtual Tokamak' [16], a web-game [17] in the educational IPPEX (Interactive Plasma Physics Experience) website, created by the Princeton Plasma Physics Laboratory, where users are allowed to configure the shape and make-up of the reactor (or 


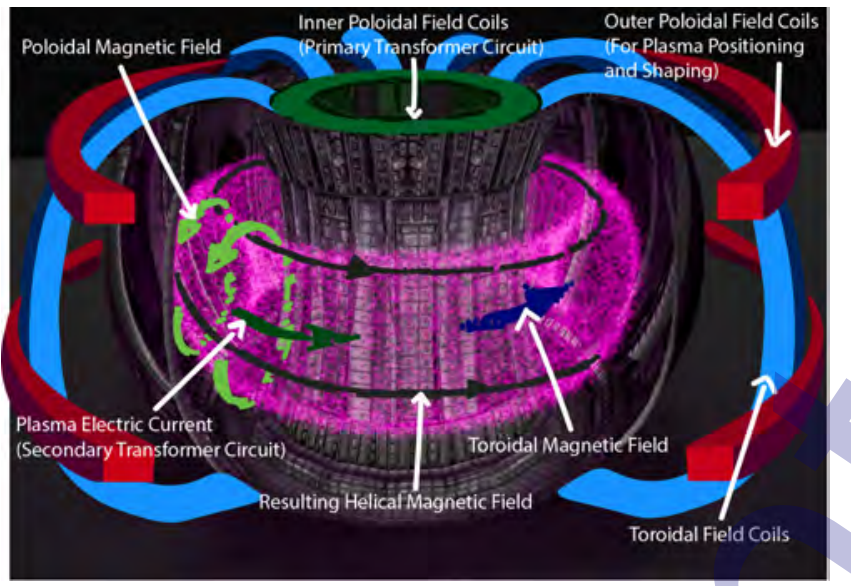

Fig. 1. The electromagnetic coils of a tokamak device, used for controlling the plasma, and the electromagnetic fields they create.

to select from preset configurations for existing tokamaks) and then tasked with manipulating three reactor controls, namely magnetic field strength, power input (for plasma heating) and plasma density (the amount of deuterium and tritium in the reactor chamber). The latter two examples employ a similar approach to our VPE, but are limited to 2D graphics and fewer controls for controlling the fusion experiment.

\section{A VirTUAl FUSion REACTOR FOR EXPERIMENTATION}

\section{A. Simulation Tokamak}

Around the world, there are several tokamaks in operation or under construction, e.g. in Japan, where JT-60SA (an upgraded Japan Torus-60) began operating in March 2021, then there is, more famously, ITER (International Thermonuclear Experimental Reactor), which is currently being assembled in the south of France, and in the UK, there are JET (Joint European Torus) and MAST-U (Mega Ampere Spherical Tokamak Upgrade) both operational and situated on the same site in Oxfordshire. The tokamak torus used in our VPE is based on JET [18]. The simulation design was guided using advice from subject experts, i.e. plasma physicists who are familiar with tokamaks and the set-up and control of fusion experiments. We implemented the virtual experience using Unreal Engine 4 , with the experimental plasma parameters controllable using a simple slider-based user interface. To emulate the plasma in our virtual reactor chamber we utilized the engine's Niagara particle effects system, as although the core of the plasma is invisible, "there is a pink glow around the edges because the plasma is cooler" [19].

\section{B. Simulation Design}

Designed, following advice by subject experts working with tokamak devices, our proposed VPE allows users to select one of four modes of operation ('easy', 'medium', 'hard' and 'spectator', the latter of these lacking interactive controls, simply allowing users to move through the environment and observe an automatically running plasma). The selection of the mode defines the level of complexity that the user will have to handle, meaning the number of parameters they must control to keep the plasma experiment running. At the time of writing, this project is still work in progress, with the 'hard' operating mode not yet fully implemented.

To understand the design of the simulation and its user interface a basic understanding of the processes involved in nuclear fusion experiments using tokamak devices (Fig. 1), such as JET [20], is required.

1) How To Create a Fusion Experiment: The reactor that holds the plasma in which fusion happens has at its heart a vacuum chamber. In the lead-up to the pulse (the experiment), a toroidal magnetic field that will direct the plasma around this reactor chamber is generated using the toroidal coils surrounding it (Fig. 1p. A small amount of gas consisting of a 50:50 ratio between Deuterium and Tritium ( $D T$ - two types of hydrogen isotope that are collided to create fusion) is then injected into the chamber to initiate the plasma, while the primary poloidal field coil (in the centre of the reactor) is used to induce a voltage in the plasma and the outer poloidal field coils are used to create a poloidal field null, keeping the plasma away from the chamber's walls so the ions and electrons perpetually follow the toroidal field. This leads to a snowball effect whereby more and more lone electrons and ions are created by these particles colliding with the atoms of the gas that was injected into the reactor chamber.

Having thus created the plasma, this then needs to be controlled, which can be achieved using different methods, e.g. by ramping-up the voltage and thereby increasing the plasma electrical current. This method can also be used to maintain this current, i.e. by controlling how fast it is increased, which at a certain point will reach what is called a 'flat top' [20], meaning that the plasma electric current is constant.

At this point there are two methods for controlling the plasma, both involving the Deuterium and Tritium levels.

1) Modifying the $D T$ ratio: if the levels of Tritium are reduced, fusion power reduces as well, which can be controlled using 'pellets' of frozen Deuterium and Tritium (at a temperature of around $20 \mathrm{~K}$ ) that are shot into the machine using compressed air. By selecting pellets of one or the other isotope, fusion power can be regulated.

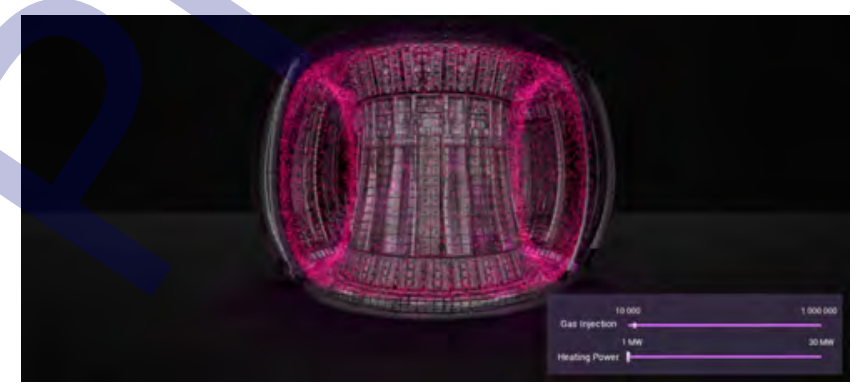

Fig. 2. Easy-Mode of the experiment. The plasma shown here fills all of the reaction chamber and the low values for the 'Heating Power' and 'Gas Injection' controls have the effect that it is relatively low-density and cool. 

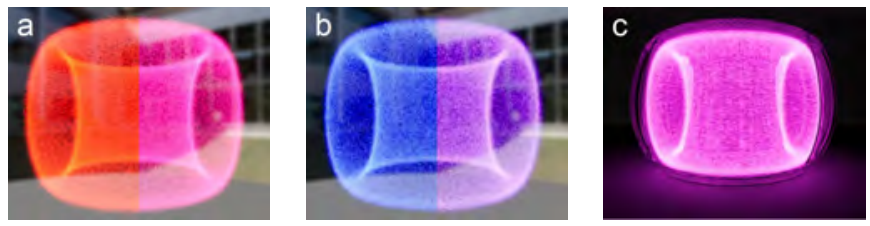

Fig. 3. Cold plasma $\left(\mathrm{a}_{\text {right }}\right)$ and hot plasma $\left(\mathrm{b}_{\text {right }}\right)$. 'Heating Power' is mapped to the particles' blue colour channel (constrained within the range 0.2 to 0.8 ) with the red channel being 1 minus the blue channel value. Fairly 'cold' plasma filling the tokamak in the non-interactive Spectator-Mode (c).

2) Modifying the heating power, employing so-called $\mathrm{Neu}$ tral Beams, i.e. beams of neutral Deuterium and Tritium. These have a significant effect on the plasma as they influence the fusion power, meaning if one stops heating the plasma, the fusion power will decrease.

2) Easy Mode: The 'Easy mode' was designed with novice users in mind, reducing the system's complexity by keeping the number of controllable parameters to a minimum (Fig. 2) to avoid overwhelming users, similar to the Nuclear Reactor Simulator [10] and slightly less complex than the IPPEX Virtual Tokamak [17], so users keep enjoying the experience while becoming acquainted with the basic principles of controlled nuclear fusion. These two parameters are 'Gas Injection', approximating controls for the density of the plasma, and 'Heating Power', representing energy input and controlling the plasma temperature. These are two of the most basic methods for controlling a plasma, by either increasing the heat of the gas or by changing its $D T$ levels.

To visualize the plasma, the input from these sliders is mapped to variables of the Niagara particle system, with 'Gas Injection' directly linked to the spawn rate of the main particles, also affecting the number of light-emitting particles at the fractional level of GasInjection / 1000. To approximate the real-world attributes of the plasma's glow, the 'Heating Power' control is mapped to the colour of the particles with the 'coldest' plasma having a reddish fuchsia colour (Fig. $3 \mathrm{a}_{\text {right }}$ ) and the 'hottest' plasma having a blueish purple tint (Fig. 33 right $_{\text {) [19]. }}$ [19.

3) Medium Mode: The 'Medium mode' is still supposed to be relatively easy to use, conveying the experience rather than an accurate portrayal of running tokamak experiments. The 'Gas Injection' and 'Heating Power' controls are identical to 'Easy mode' with the addition of two controls - slightly more complex than the Virtual Tokamak [17] - for shaping the plasma, manipulating the magnetic field confining it (Fig. 4):

1) 'Elongation' affects the height of the plasma, which in this VPE mode provides a simplified approximation of the plasma shaping controls of a real tokamak device.

2) 'Minor Radius' affects the radius of the plasma profile, i.e. the width and height of the plasma ring inside the reactor chamber.

The values that these controls can take are limited to safe values, so that their correlation - even when the controls are set to their maximum values - would allow the plasma to completely fill the reactor chamber but grow no larger, ensuring

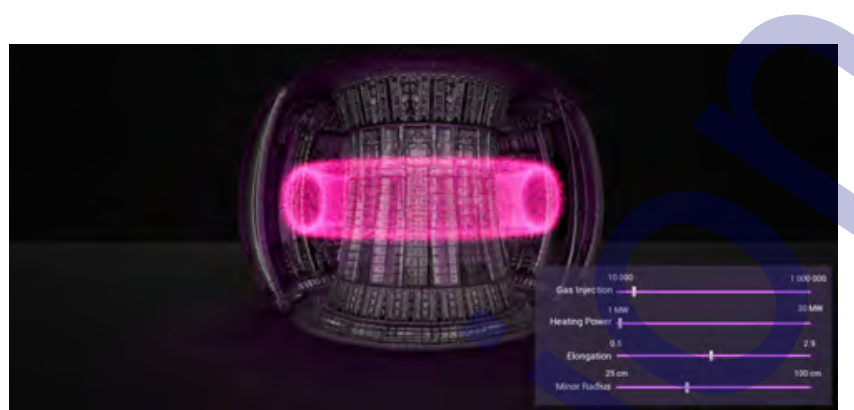

Fig. 4. Medium-Mode of the experiment. The position and shape of the plasma ring are controlled by the 'Elongation' and 'Minor Radius' controls. Low 'Heating Power' and 'Gas Injection' control values mean that the plasma shown here is relatively low-density and cool.

that the user - at this stage - cannot disrupt the plasma, losing magnetic confinement and 'crashing' the experiment, which would happen when the plasma exceeds these constraints, and in a real-life tokamak could result in damaging the device.

4) Hard Mode: The 'Hard mode' is more complex than the others, involving the application of plasma constraints that are found in real-world plasmas, e.g. regarding the ratio between plasma density and power, where if the current value of one or the other were to fall below a set threshold (based on reallife physics), then this would lead to the plasma disrupting. The controls available to the user also include more accurate plasma shaping parameters [21] and the simulation implements a further constraint based on the size of the plasma and its movement within the reactor chamber, where, if the elongation exceeded a given value, the plasma would 'run away', also leading to the plasma disrupting. If any of the thresholds is breached, resulting in a destabilization of the plasma, the user will have 10 to 20 seconds to re-stabilize the plasma before the experiment loses magnetic confinement and 'crashes', with the disruption signalled by a flash of light as a visual cue and a small bang as an audio cue, after which the user is given the choice to reset and restart the experiment.

\section{Making the Plasma Visible}

To create an interesting and engaging visual experience, the plasma had to be made visible, which is achievable through the use of a particle system. Unreal provides several means for creating particle systems, and Unreal Engine 4's Niagara subsystem proved to be ideal for implementing the plasma particle system as it allows particle systems to be confined within a torus (Fig. 4, using sprites to make the particles of the simulated plasma visible. The particle system itself is shaped to create a more accurate representation, first through the addition of a 'Vortex Velocity' module, providing the particles with a set amount of spin and also by angling the axis around which they are spinning. In 'medium mode', the 'elongation' and 'minor radius' controls map to controls that modify the height and width of the particles system representing the plasma, whereas in 'easy mode' these values are preset. 'Hard mode' will employ more accurate plasma shaping controls [21]. To prevent the particles from escaping 
the radius of the virtual tokamak reactor chamber, the particle's lifespan was decreased, balancing this with the velocity of the vortex, and noise was added to the simulation, increasing the particle system's motion and depth appearance, and visually recreating the phenomenon called zonal flows which in realworld plasmas arises from a self-organization phenomenon of the plasma. This noise also added to the stray particles appearing on the edge of the simulated plasma, emulating effects that happen in real tokamak devices, where particles hit the reactor chamber's walls. To achieve the effect of the plasma's edges glowing [19] and this glow then lighting the inside of the reactor chamber, we duplicate the plasma particle emitter, with the duplicate proportionally emitting $1 / 1000$ of the particles, which in turn are attached to evenly distributed point-light sources.

\section{WORK IN PROGRESS - DiscusSiON}

At the time of writing, our VPE has three working modes of operation, these being 'Easy' mode (section III-B2 and Fig. 22, 'Medium' mode (section III-B3 and Fig. 4) and 'Spectator' mode (Fig. 35), with the 'Hard' mode (section III-B4) still being work in progress. The development was guided by expert advice from plasma physicists and informed by current practices of using educational interactive experiences for teaching physics principles. Among the objectives that we aimed to achieve with our VPE were a degree of visual fidelity and to a certain extent - somewhat realistic functionality regarding the tokamak device as well as a visually plausible appearance of the plasma [19], and this was achieved - with the exception of the as yet unfinished 'Hard' mode.

Although unfinished, our prototype VPE has been evaluated by the experts who advised on its development, who believe "that it could be a support for teaching about fusion". A user study or evaluation to verify the prototype' efficacy for conveying the principles of nuclear fusion or plasma physics has yet to be conducted, though, the main reason for this being the COVID-19 lockdown that closed university campuses at the start of 2021, preventing access to the required facilities.

\section{CONCLUSions \& FUture WORK}

Overall, our prototype VPE - even in its incomplete state - matches the functionality of comparable applications ( [15], [16]), while adding real-time 3D visualisation, meeting these aspects of its development objectives.

Future work, apart from completion of the 'Hard mode' implementation will include an initial pilot study to get basic usability feedback from users, followed by a more exhaustive user study to evaluate the application's efficacy for the purpose that it was created for, which will hopefully be possible once the current pandemic related restrictions regarding access to the university campus are relaxed.

\section{ACKNOWLEDGMENTS}

The authors would like to thank Fernanda Rimini and Morten Lennholm for their support and advice regarding nuclear fusion and plasma physics. We are also grateful to
Valery Adzhiev and Oleg Fryazinov from the National Centre for Computer Animation, as well as to Nikolaus Muldal from the Culham Science Centre's communication department.

\section{REFERENCES}

[1] F. L. Ribe, "Fusion reactor systems," Rev. Mod. Phys., vol. 47, pp. 7-41, 1975.

[2] L. Artsimovich, "Tokamak devices," Nuclear Fusion, vol. 12, no. 2, pp. 215-252, 1972.

[3] J. E. Menard et al., "Fusion nuclear science facilities and pilot plants based on the spherical tokamak," Nuclear Fusion, vol. 56, no. 10, 2016.

[4] L. Stege, G. van Lankveld, and P. Spronck, "Teaching high school physics with a serious game," International Journal of Computer Science in Sport, vol. 11, no. 1, pp. 123-134, 2012.

[5] D. N. Carr, T. Bossomaier, and K. Lodge, "Designing a computer game to teach einstein's theory of relativity," in Computer Graphics, Imaging and Visualisation (CGIV 2007), 2007, pp. 109-114.

[6] H.-Z. S. Lin and G.-F. Chiou, "Modding commercial game for physics learning: A preliminary study," in 2010 Third IEEE International Conference on Digital Game and Intelligent Toy Enhanced Learning, 2010, pp. 225-227.

[7] H. Du, Z. Jia, J. Wang, and X. Tian, "A control mechanism of the physical simulation experiment based on game engine," in 2009 International Conference on Computational Intelligence and Software Engineering, 2009, pp. 1-3.

[8] W. Hu, J. Jiang, and L. Shi, "Electromagnetism experiment simulation based on game engine," in 2018 IEEE/ACIS 17th International Conference on Computer and Information Science (ICIS), 2018, pp. 781-785.

[9] T. Bergmann, M. Balzer, T. Hopp, T. van de Kamp, A. Kopmann, N. Tan Jerome, and M. Zapf, "Inspiration from vr gaming technology: Deep immersion and realistic interaction for scientific visualization," in Proceedings of the 12th International Joint Conference on Computer Vision, Imaging and Computer Graphics Theory and Applications IVAPP, (VISIGRAPP 2017), 2017, pp. 330-334.

[10] PlayGen, "Nuclear Reactor Simulator," Dalton Nuclear Institute Public Engagement (Schools, colleges and the public) website, The University of Manchester, 2015. [Online]. Available: http: //lotus.itservices.manchester.ac.uk:8080/

[11] P. Šianin, J. Plavšić, I. Arsenić, and M. Krmar, "Virtual reality (VR) simulation of a nuclear physics laboratory exercise," European Journal of Physics, vol. 41, no. 6, 2020.

[12] G. Stanescu, "Modern training tools in nuclear field," Rom. Journ. Phys., vol. 59, no. 9-10, pp. 989-998, 2014.

[13] J. Goncalves, T. Molto Caracena, V. Sequeira, and E. Vendrell Vidal, "Virtual reality based system for nuclear safeguards applications," in Proceedings of the IAEA Symposium on International Safeguards 2010. International Atomic Energy Agency, 2010. [Online]. Available: https://publications.jrc.ec.europa.eu/repository/handle/JRC61732

[14] J. Cham, "What is fusion," PHD Comics, Piled Higher and Deeper Publishing, 2014. [Online]. Available: http://phdcomics.com/comics. php? $\mathrm{f}=1716$

[15] Mark Film, "Operation Tokamak," Mobile App on Google Play, 2014. [Online]. Available: https://play.google.com/store/apps/details? id=dk.markfilm.operationtokamak

[16] Princeton Plasma Physics Laboratory, "Virtual Tokamak," The Interactive Plasma Physics Experience (IPPEX) website, 2020. [Online]. Available: https://ippex.pppl.gov/\#vt

[17] A. Arayath and A. Dominguez, "IPPEX Virtual Tokamak - OD Simulation Game," in 62nd Annual Meeting of the APS Division of Plasma Physics, 2020.

[18] THE JET PROJECT: Design Proposal for the Joint European Torus, EUR 5516e ed., Comission of the European Communities, March 1976.

[19] "Colour me a plasma," European Fusion Development Agreement news, November 2013. [Online]. Available: https://www.euro-fusion. org/news/detail/colour-me-a-plasma/

[20] J. Wesson, "The Science of JET," JET Joint Undertaking, Tech. Rep. JET-R(99)13, 1999.

[21] Wikipedia contributors, "Plasma shaping - Wikipedia, the free encyclopedia," 2018. [Online]. Available: https://en.wikipedia.org/w/ index.php?title=Plasma_shaping\&oldid=829085611 